\title{
WSCC 2016: the World Speed Computer Chess Championship
}

Article

Accepted Version

Krabbenbos, J., van den Herik, J. and Haworth, G. (2017) WSCC 2016: the World Speed Computer Chess

Championship. ICGA Journal, 39 (2). pp. 160-162. ISSN 13896911 Available at https://centaur.reading.ac.uk/67924/

It is advisable to refer to the publisher's version if you intend to cite from the work. See Guidance on citing.

Publisher: The International Computer Games Association

All outputs in CentAUR are protected by Intellectual Property Rights law, including copyright law. Copyright and IPR is retained by the creators or other copyright holders. Terms and conditions for use of this material are defined in the End User Agreement.

\section{www.reading.ac.uk/centaur}

\section{CentAUR}

Central Archive at the University of Reading

Reading's research outputs online 


\section{WSCC 2016: The World Speed Computer Chess Championship}

Jan Krabbenbos, Jaap van den Herik and Guy Haworth ${ }^{1}$

Amersfoort, Leiden and Reading

The six competitors in the World Chess Championship (Krabbenbos et al., 2017a) also comprised the field for the ICGA's Speed Computer Chess event, a contest emphasising the merits of good static evaluation by the engines and both reliability and hand speed from the operators. In each of the five rounds, opponents played two games, one as White and one as Black. Blitz time controls, $5^{\prime}+5^{\prime \prime} / \mathrm{move}$, were used.

Table 1

The cross-table of game scores

\begin{tabular}{cccccccccccc}
\hline$\#$ & Program & J & S & K & H & R & G & W & D & L & Score \\
\hline $\mathbf{1}$ & JONNY & & 0,0 & 1,1 & $1,1 / 2$ & $1 / 2,1 / 2$ & 1,1 & 5 & 3 & 2 & $61 / 2$ \\
$\mathbf{2}$ & SHREDDER & 1,1 & & $1,1 / 2$ & $1 / 2,1 / 2$ & $1 / 2,1$ & $1 / 2,0$ & 4 & 5 & 1 & $61 / 2$ \\
$\mathbf{3}$ & KOMODO & 0,0 & $0,1 / 2$ & & $1 / 2,1$ & 1,1 & $1,1 / 2$ & 4 & 3 & 3 & $51 / 2$ \\
$\mathbf{4}$ & HIARCS & $0,1 / 2$ & $1 / 2,1 / 2$ & $1 / 2,0$ & & $1,1 / 2$ & $1 / 2,1$ & 2 & 6 & 2 & 5 \\
$\mathbf{5}$ & RAPTOR & $1 / 2,1 / 2$ & $1 / 2,0$ & 0,0 & $0,1 / 2$ & & 1,1 & 2 & 4 & 4 & 4 \\
$\mathbf{6}$ & GRIDGINKGO & 0,0 & $1 / 2,1$ & $0,1 / 2$ & $1 / 2,0$ & 0,0 & & 1 & 3 & 6 & $21 / 2$ \\
\hline
\end{tabular}

Table 2

Opponents, game scores, accumulated scores and ranking, round by round

\begin{tabular}{|c|c|c|c|c|c|c|c|c|}
\hline \# & Program & 1 & 2 & 3 & 4 & 5 & WD L & Score \\
\hline 1 & JONNY & S: $0,0: 0,5=$ & $\mathrm{R}: 1 / 2,1 / 2: 1,5=$ & G: $1,1: 3,4$ & H: $1,1 / 2: 41 / 2,3$ & $\mathrm{~K}: 1,1: 6^{1 / 2}, 1=$ & $\begin{array}{lll}5 & 3 & 2\end{array}$ & $61 / 2$ \\
\hline 2 & SHREDDER & $\mathrm{J}: 1,1: 2,1=$ & G: $1 / 2,0: 21 / 2,2$ & $\mathrm{~K}: 1,1 / 2: 4,1=$ & $\mathrm{R}: 1 / 2,1: 51 / 2,1=$ & $\mathrm{H}: 1 / 2,1 / 2: 61 / 2,1=$ & 45 & $61 / 2$ \\
\hline 3 & KOMODO & $\mathrm{R}: 1,1: 2,1=$ & $\mathrm{H}: 1 / 2,1: 31 / 2,1$ & S: $0,1 / 2: 4,1=$ & G: $1,1 / 2: 5^{1 / 2}, 1=$ & $\mathrm{J}: 0,0: 51 / 2,3$ & 4 & $5^{1 / 2}$ \\
\hline 4 & HIARCS & G: $1 / 2,1: 1 \frac{1 / 2,3}{3}$ & $\mathrm{~K}: 1 / 2,0: 2,3=$ & R: $1,1 / 2: 31 / 2,3$ & $\mathrm{~J}: 0,1 / 2: 4,4$ & $S: 1 / 2,1 / 2: 5,4$ & 62 & 5 \\
\hline 5 & RAPTOR & $\mathrm{K}: 0,0: 0,5=$ & $\mathrm{J}: 1 / 2,1 / 2: 1,5=$ & H: $0,1 / 2: 11 / 2,6$ & $S: 1 / 2,0: 2,6$ & G: $1,1: 4,5$ & 24 & 4 \\
\hline 6 & GRIDGINKGO & $\mathrm{H}: 1 / 2,0: 1 / 2,4$ & $\mathrm{~S}: 1 / 2,1: 2,3=$ & $\mathrm{J}: 0,0: 2,5$ & $\mathrm{~K}: 0,1 / 2: 2^{1 / 2}, 5$ & R: $0,0: 2^{1 / 2}, 6$ & 136 & $2^{1 / 2}$ \\
\hline
\end{tabular}

Tables 1-3 give the cross-table, the opponents, scores and progress round by round, and the details of the games. The results most at odds with the eventual placings were SHREDDER's 2-0 round one wins over JONNY and its second round loss with White to GRIDGINKGO. JONNY continued slowly with just two draws against RAPTOR and remained in joint last place for another round. However, as if someone had thrown a switch, JONNY then scored 51/2 from 6 in the last three rounds, moving from last to first. This progress included a 20 result against KOMODO which took third place on the podium.

The games are available online (Krabbenbos et al., 2017b). Picking out some highlights, see Figures 1 and 2, one might mention first the elegant staging of a stalemate draw in the Round 2 HIARCS-KOMODO game 9

\footnotetext{
${ }^{1}$ University of Reading; g.haworth@ reading.ac.uk
} 
after 256 moves. Pawns were locked together on opposite-colour squares from the opposing bishops and the end came four moves short of a surely unavoidable draw-claim.

In game 3, with nominally equal RPP on one side, NNP on the other, JONNY exchanged the Rook for a Knight and Pawn. SHREDDER then exploited White's more exposed king to mobilise its Queen and pick up the White pawns. With colours reversed, material was also asymmetric with SHREDDER's extra piece successfully defending against JONNY's pawn majority on both wings. In Round 5 game 29, KOMODO's 33. ... Qe8? allowed JONNY to unleash a fully co-ordinated QRBN attack on Black's exposed King. KOMODO had to capture a rook at the cost of its queen and defeat swiftly followed. In game 30, with JONNY as Black, KOMODO had to defend against JONNY's excelsior h-pawn which was eventually halted on $\mathrm{f3}$ - but only at the cost of Queen for Rook again.

In the end, JONNY drew level with the highly blitz-experienced SHREDDER to force a play-off. This it duly

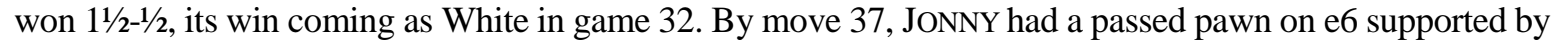
another infantryman on 55 and it may be said that this was the focus of the remainder of the game. Thirty moves later, SHREDDER had to capture the converted pawn and resignation followed.

Table 3

Games and openings, round by round

\begin{tabular}{|c|c|c|c|c|c|c|}
\hline \# & Rnd & White & Black & \multicolumn{3}{|c|}{ Res.\#m ECO Opening } \\
\hline 01 & $1 \mathrm{a}$ & GridGinkgo & Hiarcs & $1 / 2-1 / 2 \quad 48$ & E15 & Queen's Indian Defence, 4.g3 \\
\hline 02 & $1 \mathrm{~b}$ & Hiarcs & GridGinkgo & $1-0 \quad 37$ & B85 & Sicilian, Scheveningen, Classical Variation with ... Qc7 and ... Nc6 \\
\hline 03 & $1 \mathrm{a}$ & Jonny & Shredder & $0-1 \quad 103$ & D45 & Queen's Gambit Declined, Semi-Slav, 5. e3 \\
\hline 04 & $1 \mathrm{~b}$ & Shredder & Jonny & $1-0 \quad 74$ & D46 & Queen's Gambit Declined, Semi-Slav, 6. Bd3 \\
\hline 05 & $1 \mathrm{a}$ & Komodo & Raptor & $1-0$ & E12 & Queen's Indian Defence \\
\hline 06 & $1 \mathrm{~b}$ & Raptor & Komodo & $0-1$ & C80 & Ruy Lopez, Open (Tarrasch) Defence \\
\hline 07 & $2 \mathrm{a}$ & GridGinkgo & Shredder & $1 / 2-1 / 2 \quad 59$ & D46 & Queen's Gambit Declined, Semi-Slav, 6. Bd3 \\
\hline 08 & $2 \mathrm{~b}$ & Shredder & GridGinkgo & $0-1$ & E90 & King's Indian Defence, 5. Nf3 \\
\hline 09 & $2 \mathrm{a}$ & Hiarcs & Komodo & $1 / 2-1 / 2256$ & A46 & Queen's Pawn Game, Torre Attack \\
\hline 10 & $2 \mathrm{~b}$ & Komodo & Hiarcs & $1-0 \quad 61$ & D10 & Queen's Gambit Declined, Slav Defence \\
\hline 11 & $2 \mathrm{a}$ & Jonny & Raptor & $1 / 2-1 / 2 \quad 66$ & E15 & Queen's Indian Defence, 4.g3 \\
\hline 12 & $2 \mathrm{~b}$ & Raptor & Jonny & $1 / 2-1 / 2 \quad 55$ & C67 & Ruy Lopez, Berlin Defence, Open Variation \\
\hline 13 & $3 \mathrm{a}$ & GridGinkgo & Jonny & $0-1$ & D49 & Queen's Gambit Declined, Meran, 11. Nxb5 \\
\hline 14 & $3 \mathrm{~b}$ & Jonny & GridGinkgo & $1-0 \quad 106$ & E11 & Bogo-Indian Defence \\
\hline 15 & $3 \mathrm{a}$ & Hiarcs & Raptor & $1-0 \quad 59$ & B90 & Sicilian Defence, Najdorf \\
\hline 16 & $3 \mathrm{~b}$ & Raptor & Hiarcs & $1 / 2-1 / 2 \quad 38$ & B97 & Sicilian Defence, Najdorf, 7. ... Qb6 including Poisoned Pawn Var. \\
\hline 17 & $3 \mathrm{a}$ & Komodo & Shredder & $0-1 \quad 107$ & B43 & Sicilian Defence, Kan, 5. Nc3 \\
\hline 18 & $3 \mathrm{~b}$ & Shredder & Komodo & $1 / 2-1 / 2 \quad 62$ & D27 & Queen's Gambit Accepted, Classical Variation \\
\hline 19 & $4 \mathrm{a}$ & GridGinkgo & Komodo & $0-1 \quad 28$ & A 28 & English Opening, Four Knights System: $1 \ldots$. . e5 2. Nc3 Nc6 3. Nf3 I \\
\hline 20 & $4 \mathrm{~b}$ & Komodo & GridGinkgo & $1 / 2-1 / 2 \quad 28$ & A28 & English Opening, Four Knights System: $1 . \ldots$ e 5 2. Nc3 Nc6 3. Nf3 I \\
\hline 21 & $4 \mathrm{a}$ & Hiarcs & Jonny & $0-1 \quad 103$ & C67 & Ruy Lopez, Berlin Defence, Open Variation \\
\hline 22 & $4 \mathrm{~b}$ & Jonny & Hiarcs & $1 / 2-1 / 2 \quad 59$ & E04 & Catalan, Open, 5. Nf3 \\
\hline 23 & $4 \mathrm{a}$ & Raptor & Shredder & $1 / 2-1 / 2 \quad 57$ & B85 & Sicilian, Scheveningen, Classical Variation with ... Qc7 and ... Nc6 \\
\hline 24 & $4 \mathrm{~b}$ & Shredder & Raptor & $1-0$ & D45 & Queen's Gambit Declined, Semi-Slav, 5. e3 \\
\hline 25 & $5 \mathrm{a}$ & GridGinkgo & Raptor & $0-1$ & E99 & King's Indian Defence, Orthodox, Aronin-Taimanov, Main \\
\hline 26 & $5 \mathrm{~b}$ & Raptor & GridGinkgo & $1-0$ & $\mathrm{C} 83$ & Ruy Lopez, Open, Classical Defence \\
\hline 27 & $5 \mathrm{a}$ & Hiarcs & Shredder & $1 / 2-1 / 2 \quad 44$ & B49 & Sicilian, Taimanov Variation ... \\
\hline 28 & $5 \mathrm{~b}$ & Shredder & Hiarcs & $1 / 2-1 / 2 \quad 67$ & D46 & Queen's Gambit Declined, Semi-Slav, 6. Bd3 \\
\hline 29 & $5 \mathrm{a}$ & Jonny & Komodo & $1-0$ & D24 & Queen's Gambit Accepted, 4. Nc3 \\
\hline 30 & $5 \mathrm{~b}$ & Komodo & Jonny & $0-1$ & C54 & Giuoco Piano ... \\
\hline & PO a & Shredder & Jonny & $1 / 2-1 / 2 \quad 68$ & D45 & Queen's Gambit Declined, Semi-Slav, 5. e3 \\
\hline & $\mathrm{PO} \mathrm{b}$ & Jonny & Shredder & $1-0$ & D46 & Queen's Gambit Declined, Semi-Slav, 6. Bd3 \\
\hline
\end{tabular}



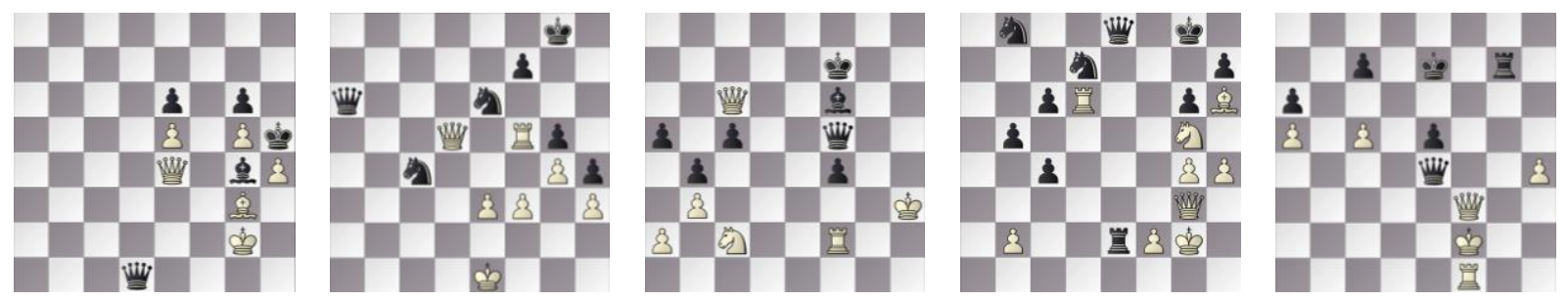

Fig. 1. a) G09 HIARCS-KOMODO 254b, b) G03 JONNY-SHREDDER 78w,

c) G04 SHREDDER-JONNY 69w, d) G29 JONNY-KOMODO 34w and e) G30 KOMODO-JONNY 55b.

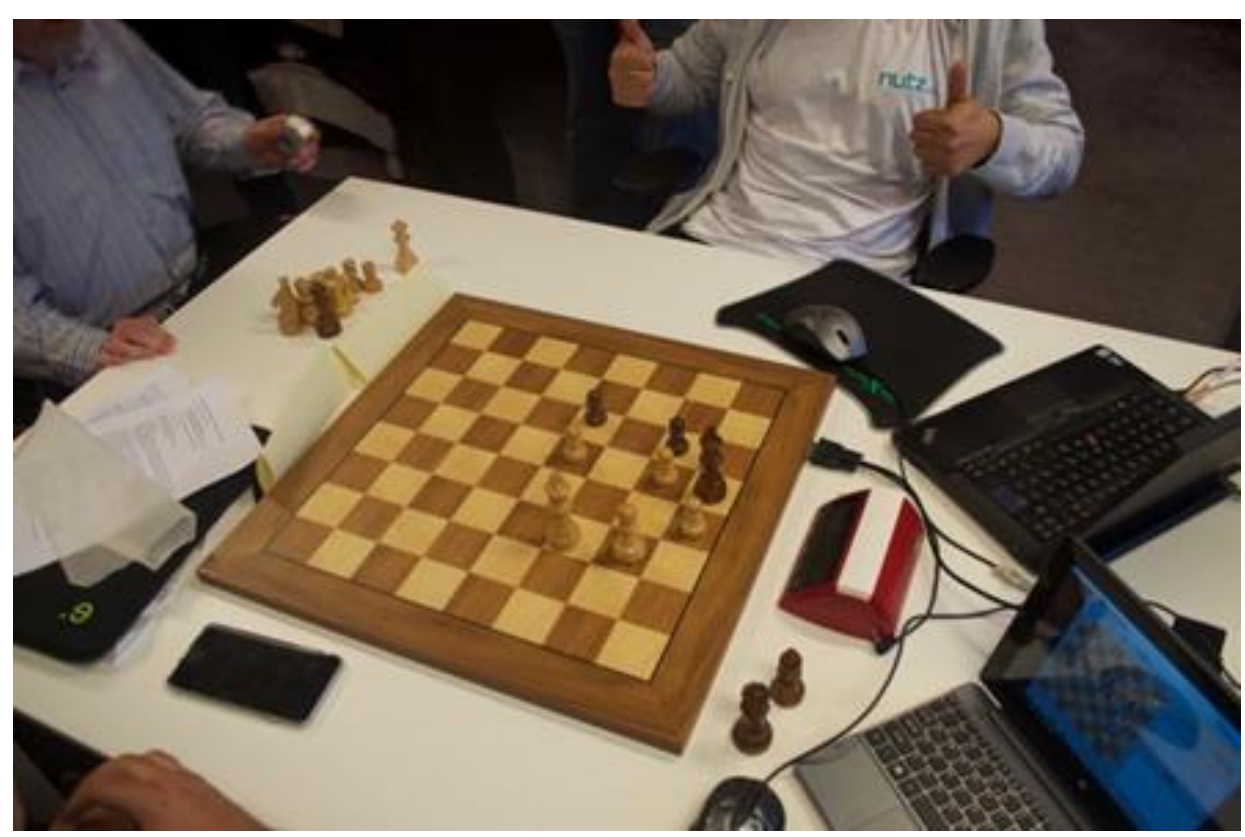

Fig. 2. Game 9: HiARCS - KOMODO, position 256b - stalemate.

Congratulations to all the participants, particularly to World Champion JONNY, SHREDDER and third-placed KOMODO, for the contest which featured many closely fought and extended games.

\section{REFERENCES}

Krabbenbos, J., van den Herik, H.J. and Haworth, G.M ${ }^{\mathrm{c} C}$. (2017a). WCCC 2016: the $22^{\text {nd }}$ World Computer Chess Championship. ICGA Journal, 39(1), 47-59. doi:10.3233/icg-170014. Pgn files available from http://centaur.reading.ac.uk/67922/.

Krabbenbos, J., van den Herik, H.J. and Haworth, G.McC. (2017b). WSCC 2016: the World Speed Computer Chess Championship. ICGA Journal, 39(2), 160-162. doi:10.3233/icg-170022. Pgn files available from http://centaur.reading.ac.uk/67924/. 